\title{
The recovery of thematic role structure during noun-noun interpretation
}

\author{
TODD R. FERRETTI \\ Wilfrid Laurier University, Waterloo, Ontario, Canada \\ and \\ CHRISTINA L. GAGNÉ \\ University of Alberta, Edmonton, Alberta, Canada
}

\begin{abstract}
We examined how people use their knowledge of events to recover thematic role structure during the interpretation of noun-noun phrases. All phrases included one noun that was a good-agent/ poor-patient ( prosecutor) in a particular event (accuse), and the other noun was a good-patient/poor-agent (defendant) for the same event. If people interpret the noun-noun phrases by inverting the nouns and applying a thematic relation (see Downing, 1977; Levi, 1978), phrases should be interpreted more easily when the head nouns typically are good agents and the modifiers are good patients for specific events. Two experiments supported these predictions. Furthermore, the results indicated that in the less preferred thematic order (agent-patient), people often generated interpretations in which the modifiers became the focus of the interpretations. This finding suggests that violating thematic role preferences is one constraint on when the inversion process occurs during noun-noun interpretation.
\end{abstract}

Thematic roles represent the semantic roles played by nouns in relation to the events denoted by verbs in sentences (Fillmore, 1968). For example, for the event arrest, cop plays the agent role, and crook plays the patient role. Earlier approaches posited that thematic role information is primarily syntactic or part of the lexical semantics of verbs and nouns. However, recent research has suggested that thematic role information includes the detailed conceptual information that people have of specific events, including the common properties of the participants and objects that are involved in these events (McRae, Ferretti, $\&$ Amyote, 1997). For example, rather than a general thematic feature, such as animacy, being the distinguishing property of the agent role of arrest, the agent role would include more detailed information, such as has the authority under the law to arrest (see also McRae, SpiveyKnowlton, \& Tanenhaus, 1998).

Given the importance of event-specific thematic role information for understanding the meaning of sentences, it should not be surprising that researchers have suggested that this information plays a role in how people interpret modifier-noun phrases. The clearest examples of how

This research was supported by an NSERC Discovery Grant and a grant from the Canadian Foundation for Innovation held by the first author, and by an NSERC Discovery Grant held by the second author. We thank Robert Nosofsky and three anonymous reviewers for helpful suggestions on this article. Correspondence concerning this article should be addressed to T. R. Ferretti, Centre for Cognitive Neuroscience, Department of Psychology, Wilfrid Laurier University, 75 University Avenue, Waterloo, ON, N2L 3C5 Canada (e-mail: tferrett@wlu.ca or christina .gagne@ualberta.ca). event-specific knowledge is used during noun phrase interpretation comes from research examining phrases that have participle modifiers (Ferretti, Gagné, \& McRae, 2003; Gagné \& Murphy, 1996; Potter \& Faulconer, 1979; Springer $\&$ Murphy, 1992). For example, Ferretti et al. examined how people integrate thematic role knowledge of agents and patients in events with the temporal and causal properties of present and past participle-noun phrases, such as arresting cop and arrested crook. As was expected, good-agent head nouns were combined more easily with present participles (arresting cop) than with past participles (arrested cop), and the reverse was true for phrases with good-patient head nouns. They also found that present-participle good-patient phrases (serving customer) were often interpreted as verb phrases, rather than as noun phrases.

In the present research, we extend the work on participantnoun phrases by examining whether thematic role information also influences the interpretation of noun-noun phrases, such as crook cop. We focus on factors that might influence the ease with which modifier-noun phrases are interpreted. The first factor concerns the use of event knowledge. We suggest that the representation of a concept involves not only information about that concept, but also knowledge about the events in which it is involved. For example, the concept teacher contains knowledge about the various events in which teachers are involved. A particularly salient event is the act of teaching. Through this event, the concept of teacher is related to the concept of student. Note that these concepts play different roles in the event: Teacher plays an agent role, whereas student plays the patient role. This knowledge might influence the ease with which a combined concept containing these concepts is interpreted. 
A second factor is the position of the agent concept in the noun phrase. There is a long held notion that "in the English language it is very common to name a thing, or express an attribute, or assert an action or manner of action by omitting minor or connecting parts of a full expression, and using only the principle elements in more or less arbitrary association and frequently in inverted order" (Teall, 1892, p. 5). For example, people can use the phrase hat box instead of box for hats. In this example, the second noun in the phrase becomes the first noun in the interpretation (i.e., the order of the nouns in the phrase is inverted in the interpretation). The notion that noun-noun compounds are derived from fuller expressions is also evident in the work of Lees (1963): "Given any two English (concrete) nouns $\mathrm{N}_{1}$ and $\mathrm{N}_{2}$, it seems always possible to find sentences of the form $\mathrm{N}_{1}-\mathrm{V}-\mathrm{N}_{2}$ " (p. 117).

One consequence of this inversion principle is that modifier-noun phrases should be easier to interpret when the ordering of the nouns is one in which the thematic role biases of the head noun are more susceptible than the modifier to being the subject of the fuller expression. ${ }^{1}$ In English, the most frequent $\mathrm{N}_{1}-\mathrm{V}-\mathrm{N}_{2}$ structure is the main clause in active voice (subject-verbed-the-object), in which the subject is most frequently an agent and the object is most frequently filled by a patient (thematically, agent-verb-patient). As a result, it should be easier to invert a modifier-noun phrase when the head noun is a good agent and the modifier a good patient for a specific event because, thematically, the order of the nouns in these phrases is consistent with the bias to invert the nouns and make the head nouns the subject of the interpretation.

\section{EXPERIMENT 1}

In Experiment 1, people provided definitions for phrases that contained two nouns that were either good agents or good patients in specific events. We manipulated the position of the agent, so that some phrases used an agent-patient ordering (e.g., cop crook) and others used a patient-agent ordering (crook cop). By examining people's definitions, we can determine whether people are more inclined to make relational interpretations when the thematic role structure of the phrase enables the goodagent noun to be easily assigned to the subject of the interpretation (i.e., patient-agent). Importantly, we can also examine the type of interpretations generated under less optimal thematic role constraints, such as when the nouns are in agent-patient order. For example, the difficulty involved in recovering and using thematic relations could lead to the generation of other forms of noun-noun interpretation, such as property (cop crook $=$ a cop prone to corruption) and conjunctive (counselor brat $=$ a brat who is a counselor) interpretations.

Another possibility is that people will still try to make event-based relational interpretations for agent-patient phrases, despite the less than optimal order of the nouns. One way that this could be accomplished and satisfy the inversion principle is to generate interpretations in the passive voice, such as the fugitive was captured by the policeman for the phrase policeman fugitive. In this case, the patient head noun is still the subject of the interpretation, and the overall meaning of the interpretation is very similar to the active form of the same interpretation. However, the active form of the main clause is far more frequent than the passive form, and so it is not clear how frequently people will make these interpretations. The second way to make relational interpretations for agent-patient phrases is to avoid inverting the nouns in the phrases when generating interpretations. That is, perhaps the strong thematic relationships between the nouns could overcome the bias to invert the nouns during interpretation, and instead, the good-agent modifiers are selected as the subject (e.g., policeman fugitive $=$ the policeman captured the fugitive). Noun-noun interpretations in which the modifier becomes the subject of interpretations, or so-called reversals, are rare, and little is known about why they occur (see Costello \& Keane, 2000, for a good discussion of this issue).

\section{Method}

Participants. Twenty participants participated for course credit. The participants in this experiment and in Experiment 2 were native English-speaking psychology undergraduates from the University of Western Ontario.

Materials. The experimental phrases consisted of 18 noun-noun pairs. The 18 pairs of nouns and their corresponding role/filler typicality ratings are listed in the Appendix. The role/filler typicality for all the nouns were determined from a previous norming study reported in McRae et al. (1997). In this study, people rated how common it was for a noun to fill either the agent or the patient role of a particular event on a 7-point scale $(1=$ very uncommon, $7=$ very common). For example, to index the agent role/filler typicality, people were asked, "How common is it for a to arrest someone?" or for the patient role they were asked "How common is it for a to be arrested by someone?" The good-agents/ poor-patients received higher agenthood ratings $(M=6.6$, range $=$ 5.8-6.9) than patienthood ratings $(M=2.4$, range $=1.4-3.9)$ $[t(17)=26.16, p<.01]$. Good-patients/poor-agents received higher patienthood ratings $(M=6.4$, range $=5.6-7.0)$ than agenthood ratings $(M=1.8$, range $=1.0-3.8)[t(17)=27.72, p<.01]$.

The 18 pairs of nouns were placed in both an agent-patient and a patient-agent order. The phrases were placed across two lists, with the restriction that each phrase appeared in both orders, no participant saw any pair of nouns more than once, and each list contained nine agent-patient phrases and nine patient-agent phrases.

Procedure. Each participant was provided with each phrase, one at a time, on a computer screen and was asked to type a definition for each phrase. The order of the phrases in each list was randomized for each participant.

\section{Results and Discussion}

The participants did not provide a response for $7 \%$ of the phrases, and another $19 \%$ of the trials involved responses that were not valid definitions. For example, responses such as both of these people are found in court for defendant prosecutor were coded as invalid because they did not explicitly provide a meaning for the phrase.

Interpretation type analysis. The valid definitions were first coded for whether they were relational (e.g., defendant prosecutor $=$ the prosecuting attorney for the 
defendant), property (crook cop $=$ a cop prone to corruption), or conjunctive (celebrity witness $=a$ witness who is a celebrity). Although the predicating relation is has been viewed as relational by other researchers (see, e.g., Levi, 1978), we included this type of interpretation in the conjunctive category, because for these items, this relation implies that the combined concept is a member of both constituent categories. Table 1 lists the percentage of each type of interpretation provided to the patient-agent and agent-patient phrases. ${ }^{2}$

The order of the agent and patient nouns in the phrases influenced the type of interpretation generated $\left[\chi^{2}(2)=\right.$ $7.14, p=.03]$. Relational interpretations were more common for patient-agent than for agent-patient phrases $\left[t_{1}(19)=2.15, p<.05 ; t_{2}(17)=2.42, p<.05 ; t_{1}=\right.$ analysis by participants, $t_{2}=$ analysis by items]. In contrast, the participants tended to generate more conjunctive interpretations for agent-patient than for patient-agent phrases $\left[t_{1}(19)=1.87, p<.08 ; t_{2}(17)=2.05, p<.06\right]$. Finally, the participants generated a similar number of property interpretations regardless of phrase order (both $t \mathrm{~s}<1$ ).

Relational interpretation analysis. Because our claims about the recovery of thematic role structure are specific to when people actually use the nouns as agents and patients in relational interpretations, we also coded the relational interpretations for whether they had this thematic structure and whether the good-agent or goodpatient nouns were the subject of the interpretations. For example, the interpretation the prosecuting attorney for the defendant was provided for the phrase defendant prosecutor. This definition was scored as an agent subject interpretation, because the subject of the interpretation was the good-agent noun in the phrase. Alternatively, the interpretation a defendant of the prosecutor was scored as a patient subject interpretation, because the subject of the interpretation was the good-patient noun in the phrase. In total, $78 \%$ of the relational interpretations involved using the nouns as either agents or patients. Interrater reliability was again calculated by having the relational interpretations scored by a second rater, who was not one of the authors. The numbers of participants who were judged to have provided agent subject and patient subject interpretations for each item were correlated. Interrater reliability was high ( $r=.99$ for agent subject interpretations; $r=$ .97 for patient subject interpretations). The percentages of these interpretations with good-agent and good-patient nouns as subjects are presented in Table 2.

Table 1

Mean Percentages of Relational, Conjunctive, and Property Interpretations in Experiment 1 (With Standard Errors)

\begin{tabular}{|c|c|c|c|c|c|c|}
\hline \multirow[b]{3}{*}{ Order } & \multicolumn{6}{|c|}{ Interpretation Type } \\
\hline & \multicolumn{2}{|c|}{ Relational } & \multicolumn{2}{|c|}{ Conjunctive } & \multicolumn{2}{|c|}{ Property } \\
\hline & $M$ & $\overline{S E}$ & $M$ & $\overline{S E}$ & $M$ & $S E$ \\
\hline Patient-agent & 50 & 4 & 18 & 4 & 9 & 2 \\
\hline Agent-patient & 37 & 5 & 27 & 5 & 7 & 2 \\
\hline Difference & +13 & & -9 & & +2 & \\
\hline
\end{tabular}

Table 2

Mean Percentages of Relational Interpretations With Agent and Patient Subjects for Both Orders of Phrases in Experiment 1 (With Standard Errors)

\begin{tabular}{cccccc}
\hline & \multicolumn{3}{c}{ Subject in Interpretation } \\
\cline { 2 - 3 } \cline { 5 - 6 } Order & \multicolumn{2}{c}{ Agent } & & \multicolumn{2}{c}{ Patient } \\
\cline { 2 - 3 } \cline { 5 - 6 } & $M$ & $S E$ & & $M$ & $S E$ \\
\hline Patient-agent & 85 & 5.2 & & 15 & 5.2 \\
Agent-patient & 34 & 8.8 & & 66 & 8.8 \\
\hline
\end{tabular}

We conducted a two-way ANOVA on the mean percentage of interpretations that had good-agent subjects. The main factor of interest was order (agent-patient vs. patient-agent), which was a within-participants and -items variable. List and item rotation group were used as between-participants and -items factors to stabilize variance due to rotating participants and items across lists in the present analysis and in Experiment 2 (Pollatsek $\&$ Well, 1995). The percentage of relational interpretations that referred to good-agent nouns was 51 percentage points higher when the order of the phrases was patientagent versus agent-patient $\left[F_{1}(1,18)=17.88, p<.001\right.$; $\left.F_{2}(1,16)=5.51, p<.05\right]$.

The results suggest that people are more likely to make relational interpretations when the head noun is a good agent, rather than a good patient. The results also show that when the head nouns are not as suitable for being the subject of relational interpretations (i.e., agent-patient phrases), people often made conjunctive interpretations instead (e.g., cop crook = a crook that is also a cop).

The analysis for agent-subject relational interpretations was consistent with the hypothesis that good agents are more likely than good patients to be assigned to the subject of the interpretations. Furthermore, when the nouns were in the less preferred thematic order, people still often generated interpretations in which the nouns were assigned to their common thematic roles. The majority of these interpretations did not involve the typical inversion of the nouns in the phrases and, instead, involved taking the good-agent modifiers as the subject (e.g., cop crook= the cop goes after the crook). As is illustrated in Table 2, people made these reversals to $34 \%$ of the agent-patient trials. Alternatively, people also assigned the nouns to their common roles by making passive interpretations (prosecutor defendant $=$ defendant questioned by the prosecutor), although this occurred for only $3 \%$ of the agent-patient relational interpretations.

\section{EXPERIMENT 2}

In Experiment 2, we used a sense-nonsense judgment task to investigate whether the ease with which a noun phrase is judged to be sensible is affected by people's knowledge about the roles that the constituents typically play in a common event. We expected people's sensitivity to thematic role knowledge associated with the nouns to facilitate judgments more when the phrases were in 
patient-agent order than when they were in agent-patient order. The strong bias to make agents, rather than the patients, the subject of the fuller expression should lead people to judge these phrases as sensible more often and more quickly than they do agent-patient phrases.

\section{Method}

Participants. Thirty participants participated for course credit.

Materials. Thirty-six phrases were created so that each noun was a typical participant for either the agent or the patient role for verbs denoting specific events (see the Appendix). Items that were good agents received higher agenthood ratings $(M=6.4$, range $=4.4$ $7.0)$ than patienthood ratings $(M=2.2$, range $=1.2-4.0)\left[t_{2}(35)=\right.$ $22.45, p<.01]$. Items that were good patients received higher patienthood ratings $(M=5.8$, range $=3.0-7.0)$ than agenthood ratings $(M=2.5$, range $=1.2-5.7)\left[t_{2}(35)=13.99, p<.01\right]$.

The pairs of nouns were placed in both an agent-patient and a patient-agent order across two lists, with the restriction that each phrase appeared in both orders and no participant saw any pair of nouns more than once. Each list also contained 36 atypical trials that were formed by combining nouns that resulted in phrases that were not very sensible ( princess plumber). Finally, 20 practice trials were constructed that included 10 sensible and 10 nonsensible phrases.

Procedure. Each trial consisted of the following events: "Ready?" was presented in the center of the computer screen until the participant pushed a button; a 500-msec blank screen was presented; then a noun phrase was presented until the participant responded. The participants were instructed to read the two-word phrase and to decide (by pushing buttons labeled "Yes" and "No") whether it had a sensible interpretation.

\section{Results and Discussion}

Latencies greater than three standard deviations above the grand mean were replaced by that value (fewer than $1 \%$ ). Response latencies for items that were judged as sensible are presented, along with percentages of sensible responses, in Table 3.

Two repeated measures ANOVAs were conducted: one with participants as a random factor and one with items as a random factor. As was predicted, patient-agent phrases were judged as sensible $181 \mathrm{msec}$ more quickly than were agent-patient phrases $\left[F_{1}(1,28)=8.56, p<.01\right.$; $\left.F_{2}(1,34)=3.77, p=.06\right]$. The percentage of sense judgments was 12 percentage points higher for patient-agent phrases than for agent-patient phrases $\left[F_{1}(1,28)=10.88\right.$, $\left.p<.01 ; F_{2}(1,34)=18.26, p<.001\right]$.

The finding that responses were faster and more accurate for patient-agent phrases than for agent-patient phrases confirms that the interpretation of modifier-noun phrases involves mapping the constituents onto a sentence-like phrase structure in which the head noun of the modifiernoun phrase becomes the subject of the fuller expression.

Table 3

Mean Response Times (in Milliseconds) and Percentages of Sensible Responses in Experiment 2 (With Standard Errors)

\begin{tabular}{|c|c|c|c|c|}
\hline \multirow[b]{3}{*}{ Phrase Type } & \multicolumn{4}{|c|}{ Dependent Measure } \\
\hline & \multicolumn{2}{|c|}{ Response Time } & \multicolumn{2}{|c|}{ Sensible Response } \\
\hline & $M$ & $S E$ & $M$ & $S E$ \\
\hline Patient-agent & 2,072 & 120 & 67 & 4.0 \\
\hline Agent-patient & 2,253 & 147 & 55 & 4.3 \\
\hline
\end{tabular}

One plausible alternative explanation for this finding is that people's familiarity with the noun-noun phrases in the different orders could account for our results if the patient-agent phrases were more familiar than were the agent-patient phrases. To investigate this possibility, we measured the familiarity of our phrases in two ways. First, we had 24 participants provide familiarity ratings for all of the noun phrases used in Experiments 1 and 2. In this task, the participants were instructed to read each phrase and judge them for how familiar they seemed to them on a scale from 1 (not familiar at all) to 7 (very familiar). In the second task, we obtained a more objective measure of familiarity of the phrases by conducting Google searches for each phrase on English Web pages. The mean familiarity ratings and the mean log Google frequencies for the agent-patient and patient-agent phrases in Experiments 1 and 2 are presented in Table 4, and the individual item values are shown in the Appendix.

For Experiment 1's items, the familiarity ratings of agent-patient phrases and patient-agent phrases did not differ $[t(17)=1.54, p>.14]$, and neither did their log Google frequencies $(t<1)$. For Experiment 2, familiarity ratings $(t<1)$ and $\log$ Google frequencies $(t<1)$ also did not differ between the two phrase types. To ensure that our results were not based on a few phrases that were rated highly familiar, we removed all phrases with mean familiarity ratings of 6 or above from the analysis in Experiments 1 and 2 (five phrases in Experiment 1, and five phrases in Experiment 2). The resulting pattern of results for both experiments did not differ in any significant way from the results reported above. Taken together, the results of the familiarity measures show that the differences observed between the two order of the phrases in Experiments 1 and 2 cannot be reduced to people's subjective feelings of familiarity or to how frequently the phrases appear in print.

\section{GENERAL DISCUSSION}

The data indicate that people use knowledge about the roles that the constituents typically play in a common event, so that phrases are more readily understood when the head noun is a good agent of the event than when the head noun is a good patient. Consequently, these results indicate that some of the same factors shown to influence thematic role assignment during sentence processing also influence noun-noun interpretation; when nouns have conceptual properties that are consistent with one role but also have properties that make them poor fillers for opposing roles, interpretation is relatively straightforward. However, as nouns become better at filling multiple thematic roles, thematic role assignment becomes more ambiguous and, thus, more difficult. ${ }^{3}$

\section{Implications for Models of Conceptual Combination}

Our results are consistent with the claims asserted by other approaches to conceptual combination. For example, the results are consistent with theories of conceptual 
Table 4

Mean Familiarity Ratings and Log Google Frequency for Both Orders of Phrases in Experiments 1 and 2 (With Standard Errors)

\begin{tabular}{|c|c|c|c|c|}
\hline \multirow[b]{2}{*}{ Phrase Type } & \multicolumn{2}{|c|}{$\begin{array}{c}\text { Familiarity } \\
\text { Rating }\end{array}$} & \multicolumn{2}{|c|}{$\begin{array}{c}\text { Log Google } \\
\text { Frequency }\end{array}$} \\
\hline & $M$ & $S E$ & $M$ & $S E$ \\
\hline \multicolumn{5}{|l|}{ Experiment 1} \\
\hline Patient-agent & 4.0 & 0.31 & 3.8 & 0.39 \\
\hline Agent-patient & 4.8 & 0.40 & 3.7 & 0.32 \\
\hline \multicolumn{5}{|l|}{ Experiment 2} \\
\hline Patient-agent & 4.0 & 0.18 & 2.7 & 0.24 \\
\hline Agent-patient & 3.9 & 0.26 & 2.7 & 0.25 \\
\hline
\end{tabular}

combination that emphasize the role of both linguistic and conceptual information during conceptual combination (see, e.g., Costello \& Keane, 2000; Gagné \& Shoben, 1997). Our results show that the syntactic structure of noun-noun phrases interacts with people's world knowledge about events to guide relational interpretations. Our results confirm that people are biased to invert the nouns in phrases and make the head noun the subject of the interpretation and then link the head noun and modifier by applying an appropriate relation. More important, our research extends these approaches by illustrating one constraint that determines whether the head nouns or the modifiers become the subject of interpretations; when the two nouns play distinct roles in specific events, people are more likely to make the head nouns the subject when they are good agents, rather than good patients. Conversely, people are more likely to make modifiers the subject when they are good agents than when they are good patients. Cases in which the modifiers are the subject of the interpretation are much more rare, and little is known about why they occur. Our results show how thematic role assignment is one constraint on why such reversals occur during relational interpretations: When the modifier is a good agent and, thus, a better subject than a good-patient head noun, people often make the modifier the subject of the interpretation.

Costello and Keane's (2000) constraint-guided approach suggests that relational interpretation occurs through linking of nouns in different roles across events that both of the nouns tend to participate in. This model suggests a crucial role for people's event-specific knowledge and even suggests that relational interpretations should be easier when nouns play more distinct roles in those events (i.e., the information associated with the nouns is more diagnostic than if they played similar roles in similar events). However, the constraint-guided approach does not specifically entail a process in which the syntactic form of the phrase interacts with peoples' world knowledge about thematic roles to influence role assignment during relational interpretation. The present results demonstrate that it is important to consider how event knowledge associated with the nouns of phrases interacts with the syntactic structure of noun-noun phrases to influence interpretation.
In conclusion, the present research suggests that when people interpret event-based combinations in isolation, they draw on knowledge about how the two nouns typically interact in events. This knowledge biases people toward thinking of a particular noun as an agent or a patient. In turn, this knowledge about agent-/patientness influences the ease of interpreting a modifier-noun phrase. By incorporating insights from research on thematic role processing during sentence processing, we can demonstrate how people recover and use thematic role information associated with nouns during conceptual combination.

\section{REFERENCES}

Costello, F., \& Keane, M. (2000). Efficient creativity: Constraintguided conceptual combination. Cognitive Science, 24, 299-349.

DownING, P. (1977). On the creation and use of English compound nouns. Language, 53, 810-842.

Ferretti, T. R., Gagné, C. L., \& McRae, K. (2003). Thematic role focusing from participle inflections: Evidence from conceptual combination. Journal of Experimental Psychology: Learning, Memory, \& Cognition, 29, 118-126.

Fillmore, C. (1968). The case for case. In E. Bach \& R. T. Harms (Eds.), Universals in linguistic theory (pp. 1-90). New York: Holt, Rinehart \& Winston.

GaGNÉ, C. L., \& MurPhy, G. L. (1996). Influence of discourse context on feature availability in conceptual combination. Discourse Processes, 22, 79-101.

GaGné, C. L., \& Shoben, E. J. (1997). Influence of thematic relations on the comprehension of modifier-noun combinations. Journal of Experimental Psychology: Learning, Memory, \& Cognition, 23, 71-87.

LEES, R. B. (1963). The grammar of English nominalizations. Bloomington: Indiana University.

LEVI, J. N. (1978). The syntax and semantics of complex nominals. New York: Academic Press.

McRae, K., Ferretti, T. R., \& Amyote, L. (1997). Thematic roles as verb-specific concepts. Language \& Cognitive Processes, 12, 137176.

McRae, K., Spivey-Knowlton, M. J., \& Tanenhaus, M. K. (1998). Modeling the influence of thematic fit (and other constraints) in online sentence comprehension. Journal of Memory \& Language, 38, 283-312.

Pollatsek, A., \& Well, A. D. (1995). On the use of counterbalanced designs in cognitive research: A suggestion for a better and more powerful analysis. Journal of Experimental Psychology: Learning, Memory, \& Cognition, 21, 785-794.

Potter, M. C., \& Faulconer, B. A. (1979). Understanding noun phrases. Journal of Verbal Learning \& Verbal Behavior, 18, 509-521.

Quirk, R., Greenbaum, S., Leech, G., \& SvartviK, J. (1985). A comprehensive grammar of the English language. London: Longman.

SPRINGER, K., \& MurPHY, G. L. (1992). Feature availability in conceptual combination. Psychological Science, 3, 111-117.

Teall, F. (1892). English compound words and phrases. New York: Funk \& Wagnalls.

\section{NOTES}

1. The head of a noun phrase is the noun that has the primary and obligatory function in the phrase. Other elements in noun phrases either "obligatory or optionally determine the head and (optionally) modify the head" (Quirk, Greenbaum, Leech, \& Svartvik, 1985). The subject of the sentence is often defined as what the sentence is about (i.e., the topic and/or focus of the sentence) and as that "which it presupposes as its point of departure" (Quirk et al., 1985).

2. Interrater reliability was calculated by having the definitions scored by a second rater, who was not one of the authors. The numbers of phrases judged as relational, property, and conjunctive for each item 
were correlated. This was not done for other interpretations, because of the numerous scores of 0 . Interrater reliability was high $(r=.90$ for relational interpretations; $r=.93$ for conjunctive interpretations; and $r=.87$ for property interpretations).

3. Although we claim that people use their knowledge of specific events to make thematic role assignments, we are not claiming that people use knowledge only about single events. Rather, we propose that because individual events are not explicitly provided, people use their knowledge about the most common roles played by each of the referents of the nouns when the two nouns are part of shared events. For some pairs of nouns, the nouns might typically tend to co-occur in a narrow range of events and might tend to play consistent roles in those events. For other pairs of nouns, the referents might commonly tend to co-occur in many events and perhaps they also tend to play multiple roles in those events.

\section{APPENDIX \\ Good-Agent and Good-Patient Nouns and Their Agenthood (Ahood) and Patienthood (Phood) Ratings for Specific Events}

FamRat and LogG refer to familiarity ratings (agent-patient/patient-agent) and log Google frequency (agent-patient/patient-agent) for each order of the phrases. Note that Items 1-18 appeared in Experiment 1 and that Items 8-43 correspond to the phrases used in Experiment 2.

\begin{tabular}{|c|c|c|c|c|c|c|c|c|}
\hline Event & Agent & Ahood & Phood & Patient & Ahood & Phood & FamRat & $\log G$ \\
\hline 1. Interview & reporter & 6.8 & 2.7 & applicant & 1.6 & 6.6 & $2.8 / 2.8$ & $2.2 / 1.1$ \\
\hline 2. Fire & owner & 6.3 & 1.9 & employee & 1.9 & 6.4 & $5.8 / 4.5$ & $5.1 / 4.5$ \\
\hline 3. Shoot & hunter & 6.9 & 2.8 & deer & 1.0 & 6.4 & $2.8 / 6.3$ & $4.2 / 6.3$ \\
\hline 4. Recognize & witness & 6.1 & 3.9 & celebrity & 3.8 & 6.8 & $2.1 / 4.3$ & $3.0 / 2.8$ \\
\hline 5. Investigate & detective & 6.4 & 1.9 & suspect & 2.4 & 6.4 & $6.4 / 2.3$ & $2.8 / 3.0$ \\
\hline 6. Slaughter & butcher & 6.2 & 1.4 & pig & 1.0 & 6.8 & $3.3 / 5.8$ & $3.0 / 4.1$ \\
\hline 7. Convict & judge & 6.4 & 1.5 & criminal & 1.4 & 5.9 & $3.8 / 4.4$ & $4.1 / 6.3$ \\
\hline 8. Accuse & prosecutor & 6.8 & 2.6 & defendant & 3.6 & 6.8 & $6.0 / 3.3$ & $3.0 / 2.8$ \\
\hline 9. Serve & waitress & 6.8 & 2.5 & customer & 1.5 & 7.0 & $5.6 / 4.3$ & $3.5 / 2.6$ \\
\hline 10. Grade & teacher & 6.9 & 2.6 & student & 2.4 & 6.8 & $6.4 / 6.5$ & $6.3 / 6.5$ \\
\hline 11. Question & lawyer & 6.5 & 2.9 & witness & 2.0 & 6.7 & $5.8 / 3.5$ & $2.9 / 2.9$ \\
\hline 12. Entertain & comedian & 6.9 & 2.7 & audience & 1.7 & 6.7 & $6.2 / 2.6$ & $2.3 / 2.6$ \\
\hline 13. Cure & doctor & 6.8 & 3.8 & patient & 1.4 & 6.1 & $6.8 / 3.1$ & $6.1 / 5.5$ \\
\hline 14. Teach & professor & 6.6 & 2.6 & trainee & 1.6 & 6.3 & $3.8 / 3.0$ & $1.7 / 2.3$ \\
\hline 15. Adopt & parent & 5.8 & 1.7 & baby & 1.4 & 6.4 & $5.9 / 3.0$ & $4.9 / 4.3$ \\
\hline 16. Invite & host & 6.5 & 2.2 & guest & 1.9 & 6.4 & $5.7 / 5.5$ & $5.2 / 5.7$ \\
\hline 17. Torture & rapist & 6.6 & 2.2 & slave & 1.3 & 5.6 & $1.6 / 2.6$ & $2.6 / 2.1$ \\
\hline 18. Arrest & cop & 6.7 & 1.6 & crook & 1.2 & 5.9 & $5.9 / 4.4$ & $3.2 / 2.4$ \\
\hline 19. Preach & evangelist & 7.0 & 1.4 & parish & 2.4 & 6.2 & $3.2 / 3.3$ & $4.4 / 2.3$ \\
\hline 20. Punish & guard & 5.0 & 2.4 & inmate & 3.8 & 5.8 & $5.2 / 4.8$ & $2.5 / 2.7$ \\
\hline 21. Punish & babysitter & 4.4 & 2.1 & child & 1.5 & 5.8 & $6.1 / 4.6$ & $3.1 / 3.0$ \\
\hline 22. Shoot & assassin & 6.9 & 2.2 & politician & 2.7 & 3.3 & $4.2 / 4.0$ & $1.3 / 2.8$ \\
\hline 23. Show & salesman & 7.0 & 1.2 & client & 2.7 & 6.4 & $2.8 / 4.2$ & $2.8 / 2.2$ \\
\hline 24. Study & archeologist & 6.8 & 1.2 & pharaoh & 2.7 & 3.0 & $1.8 / 3.0$ & $1.9 / 1.3$ \\
\hline 25. Rescue & lifeguard & 6.8 & 1.2 & victim & 2.3 & 6.6 & $2.8 / 4.7$ & $1.1 / 1.1$ \\
\hline 26. Search & investigator & 6.8 & 2.0 & suspect & 3.2 & 6.8 & $3.2 / 5.2$ & $2.5 / 3.0$ \\
\hline 27. Search & patrolman & 6.3 & 1.4 & thief & 5.7 & 6.1 & $2.2 / 2.8$ & $1.4 / 0.7$ \\
\hline 28. Capture & policeman & 6.4 & 2.1 & fugitive & 2.6 & 5.1 & $4.0 / 4.4$ & $1.0 / 1.7$ \\
\hline 29. Beat & bully & 6.5 & 1.9 & wimp & 1.7 & 4.9 & $3.0 / 5.1$ & $2.2 / 2.1$ \\
\hline 30. Deal & dealer & 6.8 & 2.2 & gambler & 5.5 & 6.6 & $3.4 / 5.0$ & $3.8 / 2.9$ \\
\hline 31. Convict & juror & 6.6 & 1.3 & criminal & 1.4 & 5.9 & $2.2 / 4.0$ & $2.3 / 2.8$ \\
\hline 32. Admire & spectator & 6.8 & 1.7 & athlete & 4.8 & 6.4 & $4.2 / 4.7$ & $2.1 / 2.4$ \\
\hline 33. Audit & accountant & 6.0 & 4.0 & taxpayer & 2.7 & 6.2 & $4.4 / 5.2$ & $2.0 / 2.5$ \\
\hline 34. Applaud & critic & 5.0 & 1.5 & musician & 2.9 & 6.8 & $2.8 / 4.1$ & $3.0 / 3.0$ \\
\hline 35. Help & counselor & 6.5 & 2.4 & brat & 2.0 & 4.0 & $2.1 / 3.0$ & $0.0 / 0.0$ \\
\hline 36. Govern & mayor & 6.8 & 1.4 & villager & 2.8 & 5.6 & $2.2 / 3.2$ & $1.5 / 2.1$ \\
\hline 37. Interrogate & inspector & 6.3 & 1.6 & culprit & 2.0 & 5.4 & $3.1 / 3.3$ & $0.3 / 1.6$ \\
\hline 38. Instruct & coach & 6.7 & 2.1 & novice & 1.6 & 5.3 & $3.8 / 3.4$ & $2.5 / 4.1$ \\
\hline 39. Evaluate & committee & 6.3 & 3.9 & applicant & 3.3 & 5.7 & $4.2 / 3.6$ & $3.0 / 2.5$ \\
\hline 40. Dismiss & principal & 6.3 & 2.0 & pupil & 1.5 & 5.5 & $2.9 / 4.3$ & $2.7 / 2.6$ \\
\hline 41. Fire & supervisor & 5.9 & 3.4 & employee & 1.9 & 6.4 & $3.7 / 6.4$ & $4.7 / 5.2$ \\
\hline 42. Execute & hangman & 5.1 & 3.9 & martyr & 2.7 & 4.0 & $2.6 / 2.5$ & $0.5 / 0.0$ \\
\hline 43. Interview & diplomat & 7.0 & 2.0 & reporter & 3.0 & 5.0 & $2.2 / 2.8$ & $1.8 / 2.3$ \\
\hline
\end{tabular}

(Manuscript received September 2, 2003;

revision accepted for publication November 14, 2005.) 